\title{
Consistency in experiments on multistable driven delay systems
}

\author{
Neus Oliver, ${ }^{1, a)}$ Laurent Larger, $^{2}$ and Ingo Fischer $^{1}$ \\ ${ }^{1}$ Instituto de Física Interdisciplinar y Sistemas Complejos IFISC (UIB-CSIC), Campus Universitat de les Illes \\ Balears, Ctra Valldemossa km 7.5, E-07122 Palma de Mallorca, Spain \\ ${ }^{2}$ FEMTO-ST, University Bourgogne Franche-Comte, CNRS, 15B Avenue des Montboucons, 25030 Besançon, \\ France
}

(Received 16 July 2016; accepted 11 October 2016; published online 27 October 2016)

\begin{abstract}
We investigate the consistency properties in the responses of a nonlinear delay optoelectronic intensity oscillator subject to different drives, in particular, harmonic and self-generated waveforms. This system, an implementation of the Ikeda oscillator, is operating in a closed-loop configuration, exhibiting its autonomous dynamics while the drive signals are additionally introduced. Applying the same drive multiple times, we compare the dynamical responses of the optoelectronic oscillator and quantify the degree of consistency among them via their correlation. Our results show that consistency is not restricted to conditions close to the first Hopf bifurcation but can be found in a broad range of dynamical regimes, even in the presence of multistability. Finally, we discuss the dependence of consistency on the nature of the drive signal. Published by AIP Publishing.

[http://dx.doi.org/10.1063/1.4966021]
\end{abstract}

Novel information processing techniques have sparked much interest recently, including the use of dynamical systems and photonic implementations of machine-learning concepts like Reservoir Computing. ${ }^{1-8}$ To achieve a successful performance of complex tasks, a property has been identified as crucial: consistency. Consistency, also referred to as reliability, is defined as the property of a nonlinear system to respond in a similar way to a repeated drive. Some studies have already investigated consistency in laser systems with delayed optical feedback and injection. ${ }^{9-11}$ However, a detailed experimental characterization of consistency in optoelectronic systems is still lacking, despite their successful utilization for Reservoir Computing. ${ }^{5}$ We employ a delay optoelectronic intensity oscillator to explore its consistency properties when external drives are applied. As a scalar system with multistability, particular dynamical features emerge under the influence of a drive. Harmonic signals and time-traces originating from the system are used as modulation or drive. The correlations indicate that a significant level of consistency can be obtained even for operation in the global chaos regime.

\section{INTRODUCTION}

In any information processing scheme, it is a crucial requirement to have a reliable operation of the system. This means assuring the same (or sufficiently similar) response of the system when tested repeatedly or, in other words, to have a well-defined relationship between the input and output. In nonlinear dynamical systems, we call this property consistency. Consistency is defined as the property of a nonlinear system to respond in a similar manner to similar inputs. ${ }^{9}$ This definition can be easily interpreted in terms of drive-

\footnotetext{
a) Author to whom correspondence should be addressed. Electronic mail: noliver@ifisc.uib-csic.es. Present address: University of Muenster, Germany.
}

response schemes, without imposing any restriction on the types of drive signals used. The boundaries between consistent and inconsistent behaviors are not yet fully defined, and the responses of a dynamical system can be classified into types of consistency. Responses that are structurally similar but not identical can still be considered consistent. But in a more restrictive scenario, we can distinguish complete consistency as the case in which the same input leads, after possible transients, to the same output of the system. ${ }^{12}$ Consistency has also been referred to as reliability and also investigated in the contexts of generalized synchronization or noise synchronization. ${ }^{13-16}$

Given its relevance, some theoretical and experimental works on consistency in nonlinear delay systems have been presented recently..$^{9-11,17} \mathrm{We}$ aim to extend the phenomenology and understanding of consistency by studying a paradigmatic nonlinear dynamical system: a delay optoelectronic intensity oscillator. Optoelectronic oscillators are hybrid systems becoming more and more important in communication technologies ${ }^{18-20}$ and have been proven to be an attractive platform for optical information processing. 5,21,22 The standard optoelectronic intensity oscillator consists of a semiconductor laser feeding a Mach-Zehnder modulator (MZM), whose output is delayed in a fiber line, detected, amplified, filtered, and finally fed back to the modulation input of the modulator.

Optoelectronic oscillators with delay exhibit a wide variety of dynamical scenarios depending on the control parameters such as feedback strength and MZM offset phase, or filter characteristics. ${ }^{23-25}$ They are known for their characteristic transition to chaos, displaying waveforms from periodic oscillations to fully developed chaos. When the employed filter is a low-pass filter, the configuration is commonly referred to as the Ikeda delayed feedback oscillator, as it was used for the investigation and characterization of features of the Ikeda delay differential equation. ${ }^{26-30}$ With a bandpass filter configuration, the optoelectronic oscillator 
shows other interesting dynamical features, including chaotic breathers $^{24}$ or chimera states. ${ }^{31}$

As a scalar nonlinear dynamical system, optoelectronic oscillators are well-studied systems in the literature, showing an excellent agreement between modeling and experiments. $22,26,28-30,32-35$ The scalar nature also allows for a recording of the response in the electrical domain and using the recorded responses for reinjection as many times as desired. Yet these systems are more constrained in terms of chaotic regimes, exhibiting only the so called weak chaos regime. ${ }^{36,37}$ Strong chaos, a major mechanism destroying consistency, does not occur in the undriven system, but in the presence of an external drive, consistency is not guaranteed. In addition, the Ikeda oscillator displays multistability and has many coexisting solutions that can jeopardize consistency. ${ }^{27,28}$ Although it has been successfully used for neuro-inspired photonic information processing implementation, in particular, Reservoir Computing, 5,38,39 successful operation has been limited to conditions close to the first instability threshold.

In this paper, we investigate the consistency properties of an optoelectronic intensity oscillator with first-order low pass filtering when subjected to an external forcing. Our analysis involves various drive signals, harmonic and those originating from the system, that lead to different responses depending on the control parameters. With our experiments, we explore the possibility to have consistent behaviour beyond the instability threshold conditions for the system with a closed loop configuration and additional drive signal.

\section{EXPERIMENT}

The optoelectronic setup used for the consistency experiments is depicted in Fig. 1. A semiconductor laser ( $\lambda=1545 \mathrm{~nm}, I_{t h}=17.9 \mathrm{~mA}$ ) is used as light source feeding a Mach-Zehnder modulator (MZM) which acts as a nonlinear element. The delay line of the loop introduces a delay of $\tau_{D}=20 \mu$ s. In Fig. 1, thin blue lines indicate the optical signal, while heavy orange lines indicate the electrical signal.
The response $v(t)$ is acquired in the electrical domain after the delay line. The external drive $w(t)$ is electrically introduced after the low-pass filter (cut-off frequency of 0.52 $\mathrm{MHz}$ ), experiencing a different electronic gain than the feedback signal $v(t)$ before being applied to the modulation electrode of the MZM. The ratio between the gains $g_{v} / g_{w}$ is approximately 0.52 . Here, the system is running in a closed loop configuration and thus influenced by delay dynamics during the addition of the drive.

The laser intensity, controlled by its pump current, is used as a control parameter when analyzing the transition to chaos, and the relevant Ikeda Hopf bifurcation threshold $\left(I_{H}\right)$ is found for a laser pump current of $25 \mathrm{~mA}$ (when operating with a MZM bias at the maximum slope for the MZM modulation transfer function). From the Hopf bifurcation threshold and the solitary lasing threshold, one can derive the normalized $\beta$ bifurcation parameter: $\beta=\frac{I-I_{t h}}{I_{H}-I_{t h}}$. The amplitude of the drive signals is usually referenced to the half-wave voltage $V_{\pi}$ of the system, which is measured to be $360 \mathrm{mV}$. The standalone device, without considering the electronic amplifier from the system input to the MZM, has a $3.9 \mathrm{~V}$ half wave voltage. Drive signals are continuously applied with an intermediate blank time ( $>600 \mu$ s, thus 30 times the delay) to let the system relax back to its autonomous dynamics. During the data acquisition, the operating point of the MZM was controlled via a DC voltage offset and set to $9.09 \mathrm{~V}$ in the experiments involving the harmonic drive. For the selfgenerated waveforms, the values used were $8.44 \mathrm{~V}$ for the periodic drive, $7.31 \mathrm{~V}$ for period-2-chaos, and $5.98 \mathrm{~V}$ for the chaotic drive. The choice of the DC voltage in every experiment was based on the bifurcation scenario. Conditions in which the bifurcation diagram remained invariant and characterized by the period doubling cascade route to chaos without hysteresis were regarded as optimal. Thus, the nominal DC voltage value was used as orientation in the experiments only. In all cases, the offset phase $\phi_{0}$ set the static operating point in the negative slope of the modulation transfer function, except for the chaotic drive, in which the dynamics

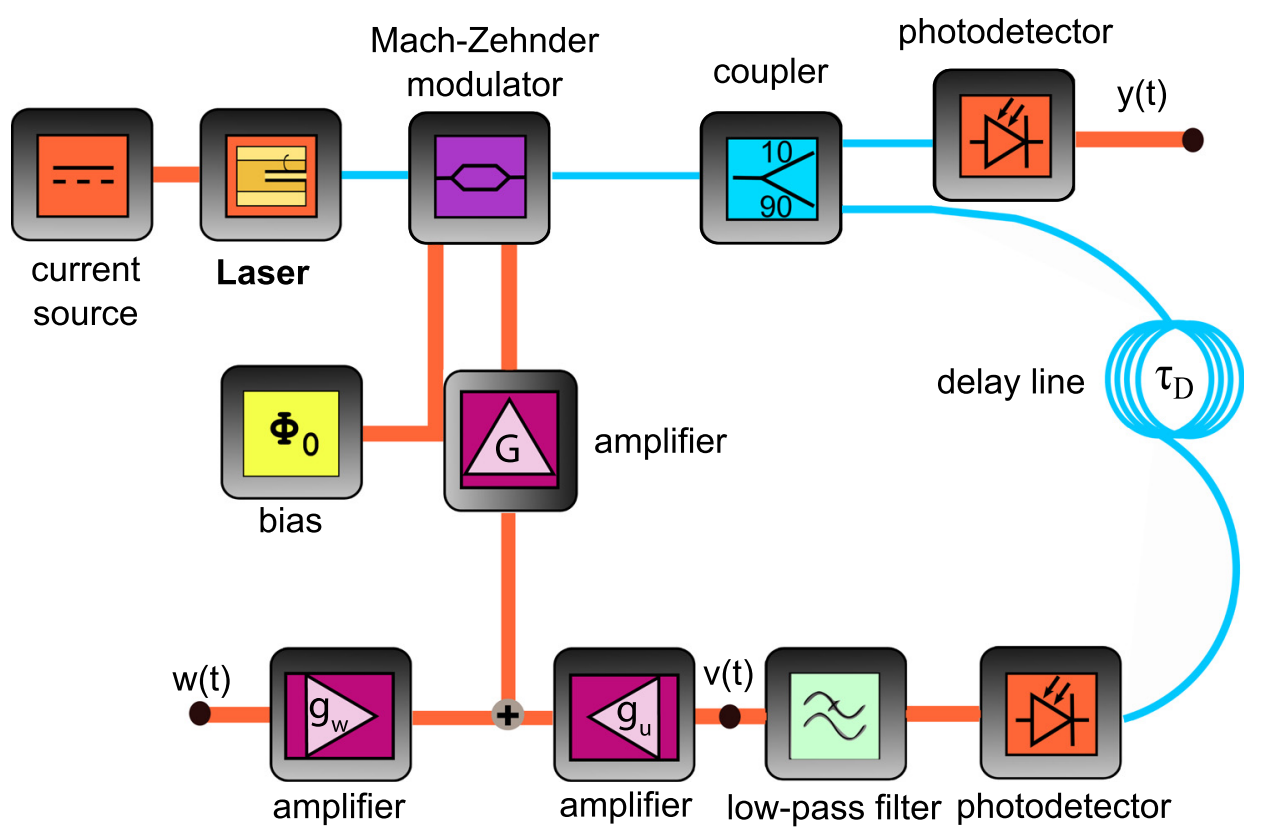

FIG. 1. Scheme of the consistency setup for the delay optoelectronic oscillator. 
were closer to the maximum of the nonlinear transformation. The multiple responses to the drive are compared, and their degree of consistency is quantified through the consistency correlation. ${ }^{9,11}$ The consistency correlation is defined as the cross-correlation coefficient between two normalized power output responses $x_{1}(t)$ and $x_{2}(t): C_{c}=\left\langle x_{1}(t) x_{2}(t)\right\rangle$, where each response is normalized such that $\left\langle x_{i}(t)\right\rangle=0$ and $\left\langle x_{i}^{2}(t)\right\rangle$ $=1, i=\{1,2\}$. Hence, the consistency correlation represents a meaningful measure that can cover the whole range from 0 to 1.

First, we discuss drive signals consisting of a harmonic signal, commonly used in other drive-response experiments, to characterize the responses of the oscillator to a smooth periodic signal. Second, self-generated autonomous dynamics are used as drives. The latter waveforms are employed to determine whether the nonlinear response of the Ikeda system is more consistent when the drive exhibits similar dynamical features as the self-generated closed-loop dynamics.

\section{RESULTS}

In the following, we analyze the responses of the optoelectronic oscillator when subjected to external inputs. The starting point of our experiments is the insertion of a harmonic drive, the frequency of which is of the order of the fast oscillations in the system. Such fast oscillations appear in the route to chaos as odd higher harmonic solutions with period $\tau_{D} / n$, with $n$ being an odd integer. ${ }^{27}$ The use of this drive contributes to characterizing how the Ikeda system responds to simple periodic signals, and to identify characteristic features of the system like transient times. The external modulation consists of a sine waveform of $200 \mathrm{~m} V_{p p}$ amplitude, below the $V_{\pi}$ of the system. The choice of the modulation frequency of $275 \mathrm{kHz}$ is not arbitrary either. After surpassing the Hopf bifurcation's threshold, the dynamics of the oscillator shows a squarewave oscillation with a periodicity of approximately $2 \tau_{D}$, distinctive of operating points along the negative slope of the transfer function of the MZM. The fundamental frequency of the squarewaves is $25 \mathrm{kHz}$; thus, the modulation frequency corresponds to the 11th harmonic of the system. Each harmonic sequence has 80 periods and it is injected repeatedly 77 times with the blank time in between.

When no modulation is applied, the dynamics of the optoelectronic system exhibits periodic oscillations at the fundamental frequency under the chosen operating conditions. In Fig. 2(a), we show that the application of the harmonic drive results in an adapted behaviour of the system with oscillations at the drive frequency. The transient time required for the system to converge to the adapted response corresponds to about the delay time $\tau_{D}$. After that time, the system responds in the same way to every repetition of the drive, displaying consistency among responses. When the modulation is switched off, the system goes back to the original oscillations of smaller amplitude at $25 \mathrm{kHz}$.

A different behaviour is observed when the dynamical conditions are changed by choosing a pump current of $\mathrm{I}=37.03 \mathrm{~mA}$. The autonomous system is then in a period-2-

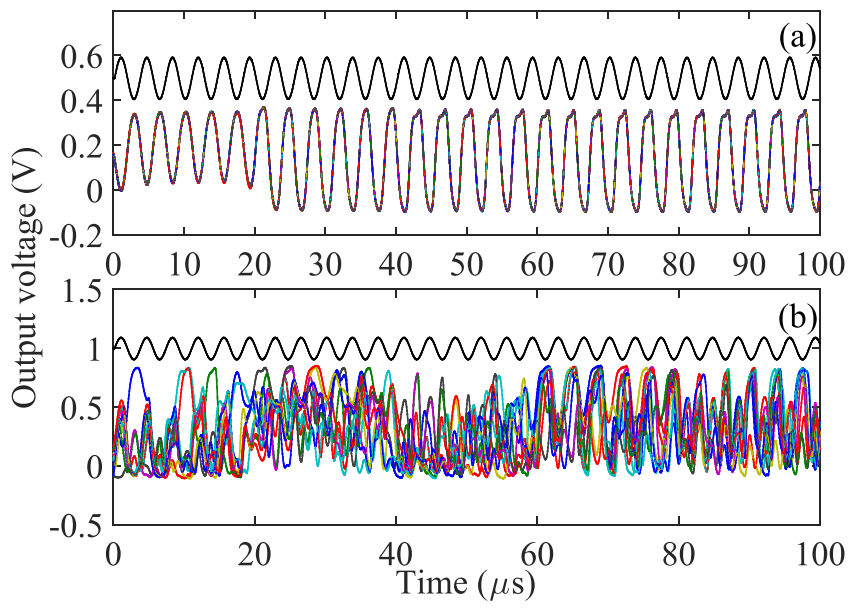

FIG. 2. (a) Experimental time traces under modulation for a pump current of $\mathrm{I}=26.93 \mathrm{~mA}$; (b) experimental time traces under modulation for $\mathrm{I}=37.03 \mathrm{~mA}$. The sinusoidal drive is also represented in black color with an offset to be distinguishable from the waveforms.

chaos state. As illustrated in Fig. 2(b), the time traces exhibit periodic oscillations of the same frequency as the drive, but with irregular variations in amplitude. In general terms, the oscillation phase is preserved among the different trials, and the range of the oscillations is clearly bounded. This example represents the situation of a low (partial) degree of consistency. Moreover, the system requires a longer transient time of $80 \mu$ s to display a consistent oscillation phase.

To quantify the degree of consistency, we compute the consistency correlation. The results are shown in Fig. 3 as a function of the pump current. The dotted vertical lines divide the dynamical regimes of the autonomous system though, given the jumps between multiple stable solutions, strict boundaries cannot be defined. Various realizations with different offset phases $\phi_{0}$ show that the trend of the curve is more or less invariant of the offset phase $\phi_{0}$. When the system is in a steady state or periodic oscillations regime, the

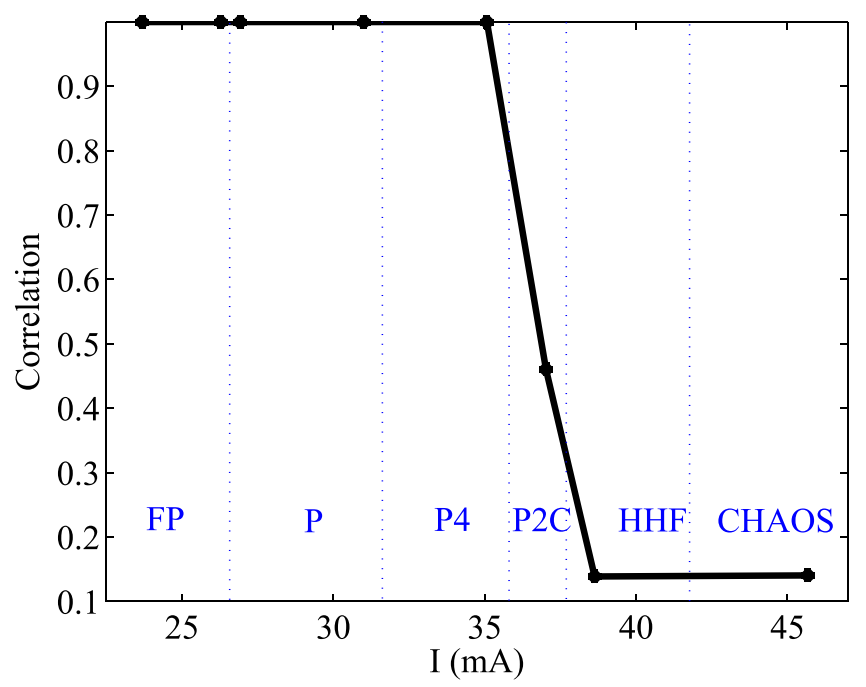

FIG. 3. Correlation as a function of the pump current I for an harmonic modulation of $200 m V_{p p}$. The dotted lines divide in an approximate manner the different dynamical regimes found at different I. FP: Fixed Point; P: Periodic, P4: Period 4; P2C: Period-2-chaos; HHF: Higher Harmonic dynamics; and Chaos. 
response to a harmonic forcing is consistent; the system adapts easily to the modulation as the high values of the consistency correlation indicate. When the system enters into the chaotic regime, the response is not consistent anymore and the correlations drop drastically. However, a regime in which a gradual transition between consistent and inconsistent response occurs can be identified. In this regime, the autonomous dynamics is within the period doubling sequence, like period 4 , or even period-2-chaos, and the corresponding pump currents fall within the range of (32-37.5) mA. In this transition area, the system preserves partially its autonomous dynamics while displaying influence from the modulation frequency. The results summarized in Fig. 3 are distinctive for the case in which the modulation frequency corresponds to a higher harmonic solution. Operating in the vicinity of fixed point or periodic autonomous dynamics, the response system is influenced by the drive and adjusts its frequency to the external one. However, as soon as chaos is present in the autonomous dynamics, consistency drops.

Next, we repeat the procedure employing more complex drive signals. Instead of harmonic waveforms, we now use time traces generated by the autonomous system (running in a closed loop configuration), recorded by a digital scope and then replayed as inputs using an arbitrary waveform generator. The purpose is to investigate whether the system reacts differently to these drives so that even in the chaotic regimes forms of consistency can be identified.

Three time traces were acquired at different operating conditions to be used as drives. Specifically, the waveforms correspond to the periodic, period-2-chaos, and fully developed chaos regimes of the Ikeda optoelectronic system. The period2-chaos belongs to a regime of local chaos, in which the periodic solutions merge into chaotic attractors but the dynamics still exhibits periodic structures. Eventually, the chaotic attractors merge again into a single attractor, leading to the fully developed chaos state with no apparent structures. ${ }^{28}$ The periodic waveform was recorded for $\mathrm{I}=30.01 \mathrm{~mA}$, with a natural amplitude of $V_{0}=0.6 V_{p p}$. This case study can be seen as an extension of the harmonic drive investigations with different periodic waveform and a slower frequency of $50 \mathrm{kHz}$. The period-2-chaos drive was acquired at $\mathrm{I}=37.57 \mathrm{~mA}$ and has an original amplitude of the oscillations of $0.97 V_{p p}$, similar to the amplitude of the chaotic oscillations exhibiting $1 V_{p p}$ (acquired at $\mathrm{I}=49.81 \mathrm{~mA}$ ). The drive signals $w(t)$ were injected into the system preserving their original amplitude as recorded. Nevertheless, we also explore the dependence of the consistency properties on the drive amplitude by attenuating the signals down to $20 \%$ of the original amplitude.

For every drive signal, and analogously to the harmonic case, we tuned the pump current to obtain data for 6 different conditions corresponding to different dynamical regimes, which were selected manually to avoid operating conditions too close to bifurcations. Those regimes correspond to fixed point, periodic dynamics, period 4 dynamics, period-2-chaos dynamics, higher harmonic frequency dynamics and global chaos. Here, the duration of the time traces used for modulation were $10 \mathrm{~ms}$ (ca. 500 times $\tau_{D}$ ), and the blank times lasted $4 \mathrm{~ms}$ (ca. 200 times $\tau_{D}$ ).

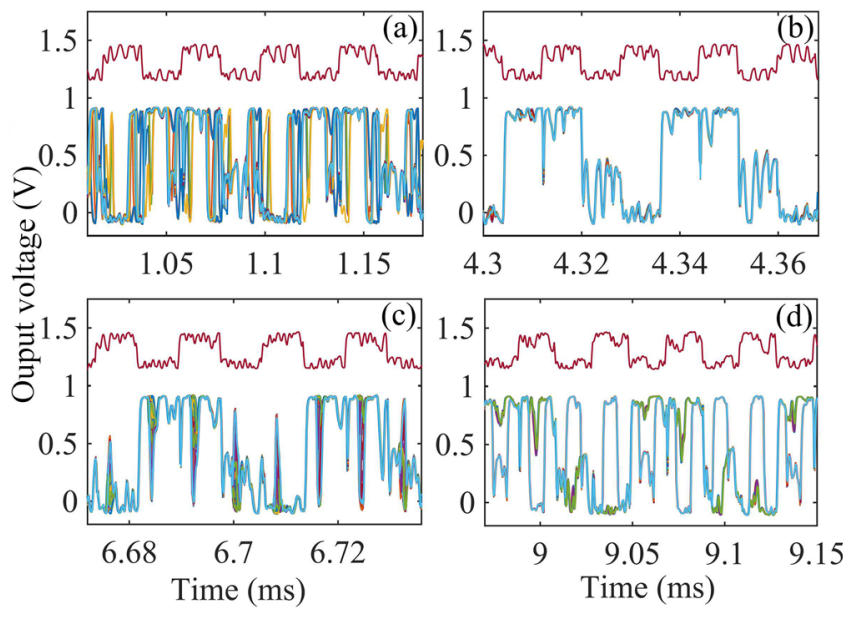

FIG. 4. Modulation drive corresponding to period-2-chaos (in red with an added offset) and 139 superimposed dynamical responses for different time intervals along the sequence, displaying (a) least consistent behavior; (b) complete consistency; (c) partial consistency; and (d) bistability.

Some examples of the dynamical responses are shown with their respective drives in Figures 4-6. Figure 4 depicts the response dynamics of the system to the period-2-chaos modulation at different times. The drive signal was injected into the optoelectronic system at a pump current of $\mathrm{I}=39.05 \mathrm{~mA}$, corresponding to a period-2-chaos or higher harmonic autonomous dynamics. The modulation amplitude, plotted in red with an offset for better visualization, was attenuated by $50 \%$ in the experiment. Below the modulation, a superposition of 139 response waveforms is depicted for different time intervals of similar duration. Figure 4(a) shows the least consistent dynamics, in which the multiple repetitions of the drive result in different trajectories, with nevertheless some similarities. A transition to high consistency is achieved after $3.5 \mathrm{~ms}$, as illustrated in plot (b). In this case, a perfect synchronized response is reached for all the 139 responses. This consistent behavior is sustained for more than $5 \mathrm{~ms}$ (corresponding to about 300

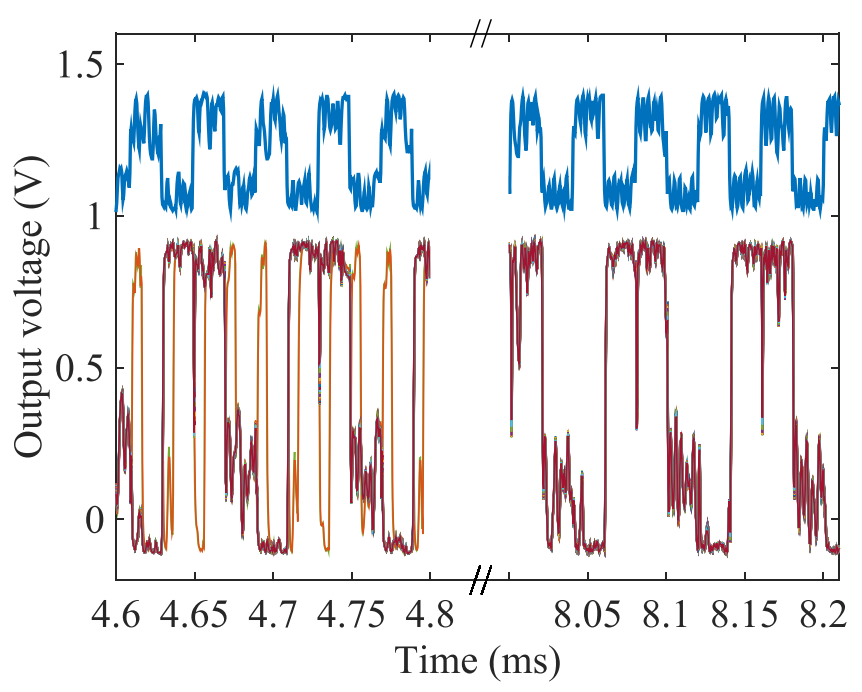

FIG. 5. Modulation drive corresponding to period-2-chaos (in blue with an offset) and 140 superimposed dynamical responses with a break in the time axis to illustrate the restoration to complete consistency. The drive signal was injected into the optoelectronic oscillator at a pump current of $\mathrm{I}=39.05 \mathrm{~mA}$. 


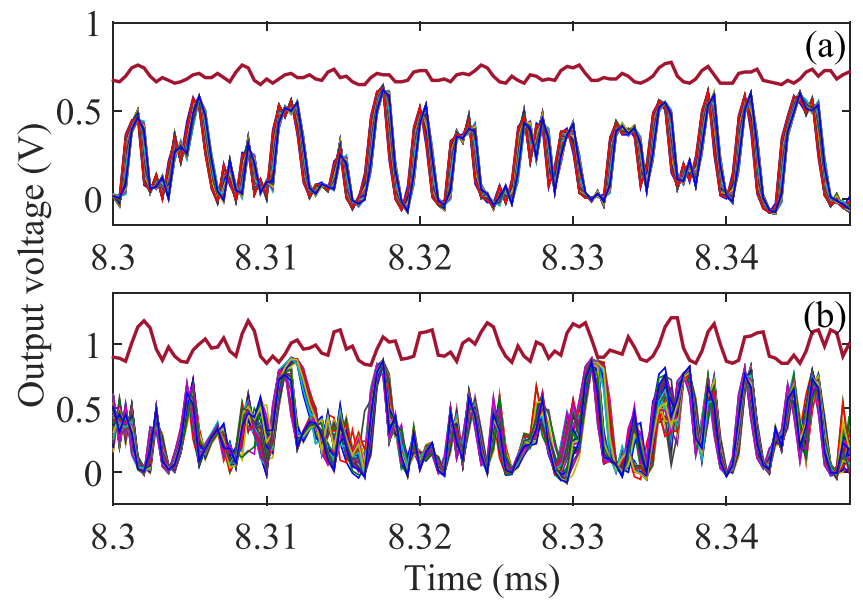

FIG. 6. Chaotic drive (red) and 50 dynamical responses for (a) $0.2 V_{p p}$ at period 4 dynamics $\mathrm{I}=34.98$. (b) $0.8 V_{p p}$ at higher harmonic dynamics $\mathrm{I}=39.58 \mathrm{~mA}$

delay times $\tau_{D}$ ), although intervals with lower degree of consistency appear, like in plot (c). This loss of complete consistency leads to a gradual change in the dynamics that ends up in bistability and oscillations of higher frequency, as plot (d) illustrates. The 139 dynamical responses are encompassed into two possible trajectories, and surprisingly, the bistability is observed during the modulation of the system and not right after the start or removal of the drive. The whole sequence of responses goes through a process of transitory dynamics, low consistency, complete consistency, and bistability, and it should be noted that every transition happens at a precise and consistent timing along the many repetitions.

Interestingly, the temporal changes in the dynamical responses are not limited to a degradation of the consistency. The opposite situation, in which consistency is restored, has also been observed. Figure 5 displays the restoration of complete consistency in the responses to the period-2-chaos drive attenuated by $40 \%$. The drive is depicted in blue with an offset of $1.2 \mathrm{~V}$ together with 140 superimposed dynamical responses. The trials exhibit a bistable behaviour in their trajectories for approximately $6.2 \mathrm{~ms}$. After that time, a complete consistency is restored and preserved until the removal of the drive. These phenomena represent a new and not yet reported form of temporal-dependent consistency. Such temporal consistent switching could be due to the vicinity of coexisting attractors, but the detailed mechanisms leading to this behaviour are still to be investigated.

Two other examples of the driven dynamics for the chaotic drive are depicted in Fig. 6. Plot (a) shows the modulation in red color attenuated to $200 \mathrm{~m} V_{p p}$ and with a manual offset together with 50 dynamical responses obtained at $\mathrm{I}=34.98 \mathrm{~mA}$. Plot (b) shows 50 responses to the same drive with an amplitude of $0.8 V_{p p}$ and acquired at $\mathrm{I}=39.58 \mathrm{~mA}$. Two important features are worth noting. In both cases, all the trials overlap in fluctuations that differ from the drive, which demonstrates the ability of the system to respond nonlinearly and consistently to the modulation. Less deviations among responses and, thus, higher consistency, are found in Fig. 6(a), which corresponds to the smaller modulation amplitude and lower pump current. A comparison between the dynamics of plots (a) and (b) also illustrates that the responses are characteristic to the operating conditions, given that, although the same drive is employed, the responses display different oscillations.

To quantify the consistency behaviour for the selfgenerated drives, we again determine the consistency correlations. Figure 7 shows the consistency correlation coefficients for the three employed drives for different dynamical conditions tuned by the laser intensity. We show two representative drive amplitudes for each signal: $200 \mathrm{~m} V_{p p}$ and $600 \mathrm{~m} V_{p p}$, so that one is below and one is above $V_{\pi}$ of the system. The consistency correlation for the periodic waveform sketched in blue color is closest to the harmonic case, with high correlation values for small pump currents and a monotonic decrease as the pump current is increased. The response system follows the drive for low pump currents, but when entering in the chaotic regime, consistency cannot be sustained. For higher amplitude of the drive (blue dashed line), the consistent response is extended to larger pump current values, allowing the occurrence of a consistent behavior in dynamical conditions beyond periodic oscillations. The green lines show the consistency correlations for a period-2chaos drive. The low amplitude case shows a higher consistency with respect to the periodic drive, extending the range of partially consistent responses. An interesting extension is the consistency correlation curve for $600 \mathrm{~m} V_{p p}$ amplitude (dashed green line). The consistent behaviour is sustained up to pump currents around $39 \mathrm{~mA}$, exhibiting correlations above 0.9 in regimes of local chaos. By increasing the pump current further, responses with a high degree of consistency can still be achieved. This is remarkable and might be related to the matching of dynamical bandwidth between the drive and response system. The correlation curves for the chaotic drive are plotted in magenta. The solid line shows the performance for the low amplitude. With this drive, we obtained

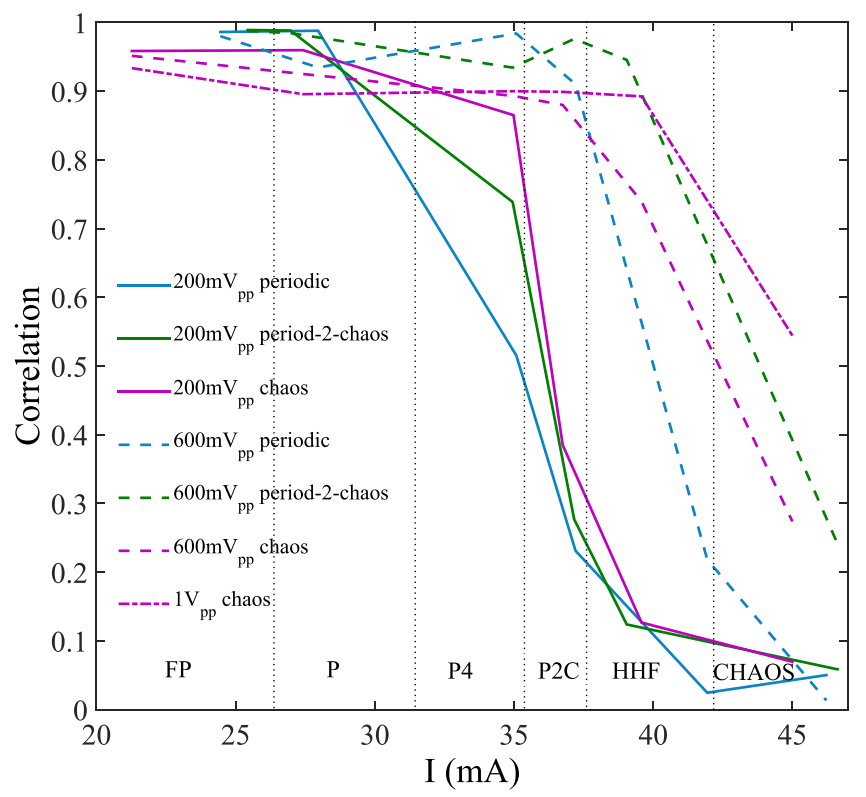

FIG. 7. Consistency correlation as a function of the pump current I for three self generated drives and two amplitudes, $200 m V_{p p}$ and $600 m V_{p p}$. The dotted vertical lines divide the different dynamical regimes found at different I. FP: Fixed Point; P: Periodic, P4: Period 4; P2C: Period-2-chaos; HHF: Higher Harmonic dynamics; and Chaos. 
the most consistent responses in intermediate dynamical regimes like the period 4 dynamics, period-2-chaos, or higher harmonic dynamics. For the case of $600 \mathrm{~m} V_{p p}$, the high correlation values at moderate and low pump currents are extended, and the decreasing slope is less pronounced as compared to the previous results. For completeness, Fig. 7 also displays the case of the chaotic drive without attenuation $\left(1 V_{p p}\right)$. Surprisingly, consistent responses are obtained in most of the regimes, showing only a gradual decay in the consistency correlations when the system is entering the fully developed chaos regime. Such observation can be also understood as the occurrence of generalized locking between the autonomous system dynamics and a structurally similar drive, although the detailed conditions for this phenomenon to arise remain to be identified. Nevertheless, these are promising results in terms of the response properties of the optoelectronic oscillator, and they prove the potential of the system to extend its consistent behaviour for information processing applications beyond the first Hopf bifurcation. Altogether, two important features can be extracted from Fig. 7. First, the amplitude of the drive can have an impact on the consistency in the regimes of period-4, period-2chaos, or higher harmonics. Larger drive amplitudes in these regimes lead to higher consistency correlations, thus extending the consistent behaviour to higher pump currents. Second, the results demonstrate that our oscillator responds with a higher level of consistency when the drive is more similar to the intrinsic operating conditions.

\section{CONCLUSION AND OUTLOOK}

We investigated the consistency characteristics of a delay optoelectronic oscillator subject to different external drive signals: harmonic drives and recorded waveforms. The drives were applied to the Mach-Zehnder modulator while the system was in closed-loop operation and in different dynamical regimes. Our results show, in general terms, that a highly consistent response is attainable when the system is operating in the periodic regime, regardless of the drive or its amplitude. As the pump current of the laser is increased, the system is more sensitive to external perturbations and consistency strongly depends on the operating conditions and the drive properties.

The harmonic drive illustrates how the consistency correlation curve for the responses exhibits a decrease as the dynamics starts to exhibit chaotic characteristics. Interesting results are also observed when signals generated by the system are used for driving with varying amplitudes. Although the trend in correlations is similar, the oscillator exhibited different forms and levels of consistency. Multistability can destroy consistency by switching between dynamical states in the course of the driving process or at the recovery times. But then, such bistability also leads to another interesting manifestation of consistent behaviour: the occurrence of the switch from one solution to another during the application of the drive at the same instant, which we named temporally consistent switching.

For almost all drive signals, the responses still preserve partial consistency when the system operates in a regime of local chaos. The amplitude of the drive influences the start of the decay in the consistency correlation as the system enters into the regime of global chaos. In the example of the chaotic drive, the degree of consistency is even sustained for fully developed chaos. These results illustrate the potential of the driven Ikeda system and open new perspectives for information processing techniques, which could extend their performance beyond the first Hopf threshold bifurcation. From a more fundamental point of view, it is an interesting question to explore, whether the consistency transition can be described in terms of a phase transition. Moreover, comparing the observed features to the brain, which is permanently in a complex dynamical state, it might be interesting to extend and understand neuro-inspired information processing concepts in the context of such conditions.

\section{ACKNOWLEDGMENTS}

This work was supported by CSIC via the PIE project (reference 201450E061), Labex ACTION (ANR-11-LABX01-01), and ANR project BiPhoProc (ANR-14-OHRI-000202). N.O. was funded by a JAE-PreDoc grant and the program Estancias Breves from CSIC and cofounded by Programa Operativo FSE 2007-2013.

${ }^{1}$ W. Maass, T. Natschläger, and H. Markram, "Real-time computing without stable states: A new framework for neural computation based on perturbations," Neural Comput. 14(11), 2531-2560 (2002).

${ }^{2}$ H. Jaeger and H. Haas, "Harnessing nonlinearity: Predicting chaotic systems and saving energy in wireless communication," Science 304(5667), 78-80 (2004)

${ }^{3}$ K. Vandoorne, W. Dierckx, B. Schrauwen, D. Verstraeten, R. Baets, P. Bienstman, and J. Van Campenhout, "Toward optical signal processing using photonic reservoir computing," Opt. Express 16(15), 11182-11192 (2008).

${ }^{4}$ L. Appeltant, M. C. Soriano, G. Van der Sande, J. Danckaert, S. Massar, J. Dambre, B. Schrauwen, C. R. Mirasso, and I. Fischer, "Information processing using a single dynamical node as complex system," Nat. Commun. 2, 468 (2011).

${ }^{5}$ L. Larger, M. C. Soriano, D. Brunner, L. Appeltant, J. M. Gutierrez, L. Pesquera, C. R. Mirasso, and I. Fischer, "Photonic information processing beyond Turing: an optoelectronic implementation of reservoir computing," Opt. Express 20(3), 3241-3249 (2012).

${ }^{6}$ Y. Paquot, F. Duport, A. Smerieri, J. Dambre, B. Schrauwen, M. Haelterman, and S. Massar, "Optoelectronic reservoir computing," Sci. Rep. 2, 287 (2012).

${ }^{7}$ F. Duport, B. Schneider, A. Smerieri, M. Haelterman, and S. Massar, "Alloptical reservoir computing," Opt. Express 20(20), 22783-22795 (2012).

${ }^{8}$ D. Brunner, M. C. Soriano, C. R. Mirasso, and I. Fischer, "Parallel photonic information processing at gigabyte per second data rates using transient states," Nat. Commun. 4, 1364 (2013).

${ }^{9}$ A. Uchida, R. McAllister, and R. Roy, "Consistency of nonlinear system response to complex drive signals," Phys. Rev. Lett. 93(24), 244102 (2004).

${ }^{10}$ G. Giacomelli, S. Barland, M. Giudici, and A. Politi, "Characterizing the response of chaotic systems," Phys. Rev. Lett. 104(19), 194101 (2010).

${ }^{11}$ N. Oliver, T. Jüngling, and I. Fischer, "Consistency properties of a chaotic semiconductor laser driven by optical feedback," Phys. Rev. Lett. 114, 123902 (2015).

${ }^{12} \mathrm{~T}$. Jüngling et al., "Consistency of chaotic systems driven by time-delayed feedback," Phys. Rev. E (unpublished).

${ }^{13}$ N. F. Rulkov, M. M. Sushchik, L. S. Tsimring, and H. D. I. Abarbanel, "Generalized synchronization of chaos in directionally coupled chaotic systems," Phys. Rev. E 51, 980-994 (1995).

${ }^{14}$ H. D. I. Abarbanel, N. F. Rulkov, and M. M. Sushchik, "Generalized synchronization of chaos: The auxiliary system approach," Phys. Rev. E 53(5), 4528-4535 (1996). 
${ }^{15}$ L. Kocarev and U. Parlitz, "Generalized synchronization, predictability, and equivalence of unidirectionally coupled dynamical systems," Phys. Rev. Lett. 76(11), 1816-1819 (1996).

${ }^{16} \mathrm{~S}$. M. Wieczorek, "Noise synchronisation and stochastic bifurcations in lasers," in Nonlinear Laser Dynamics: From Quantum Dots to Cryptography, edited by K. Lüdge (Wiley-VCH Verlag GmbH \& Co. KGaA, 2011).

${ }^{17}$ J. Nakayama, K. Kanno, and A. Uchida, "Laser dynamical reservoir computing with consistency: An approach of a chaos mask signal," Opt. Express 24(8), 8679-8692 (2016).

${ }^{18}$ L. Larger, J.-P. Goedgebuer, and F. Delorme, "Optical encryption system using hyperchaos generated by an optoelectronic wavelength oscillator," Phys. Rev. E 57(6), 6618 (1998).

${ }^{19}$ A. Uchida, F. Rogister, J. García-Ojalvo, and R. Roy, "Synchronization and communication with chaotic laser systems," Prog. Opt. 48, 203-341 (2005).

${ }^{20}$ A. Argyris, D. Syvridis, L. Larger, V. Annovazzi-Lodi, P. Colet, J. GarcíaOjalvo, I. Fischer, C. R. Mirasso, L. Pesquera, and K. A. Shore, "Chaosbased communications at high bit rates using commercial fiber-optic links," Nature 438(18), 343-346 (2005).

${ }^{21}$ L. Larger and J. M. Dudley, "Nonlinear dynamics: Optoelectronic chaos," Nature 465(7294), 41-42 (2010).

${ }^{22}$ L. Larger, "Complexity in electro-optic delay dynamics: Modelling, design and applications," Philos. Trans. R. Soc. London A 371(1999), 20120464 (2013).

${ }^{23}$ V. S. Udaltsov, L. Larger, J.-P. Goedgebuer, M. W. Lee, E. Genin, and W. T. Rhodes, "Bandpass chaotic dynamics of electronic oscillator operating with delayed nonlinear feedback," IEEE Trans. Circuits Syst. 49(7), 1006-1009 (2002).

${ }^{24}$ Y. C. Kouomou, P. Colet, L. Larger, and N. Gastaud, "Chaotic breathers in delayed electro-optical systems,” Phys. Rev. Lett. 95(20), 203903 (2005).

${ }^{25}$ M. Peil, M. Jacquot, Y. K. Chembo, L. Larger, and T. Erneux, "Routes to chaos and multiple time scale dynamics in broadband bandpass nonlinear delay electro-optic oscillators," Phys. Rev. E 79(2), 026208 (2009).

${ }^{26} \mathrm{~K}$. Ikeda, "Multiple-valued stationary state and its instability of the transmitted light by a ring cavity system," Opt. Commun. 30(2), 257-261 (1979).
${ }^{27}$ K. Ikeda, K. Kondo, and O. Akimoto, "Successive higher-harmonic bifurcations in systems with delayed feedback," Phys. Rev. Lett. 49(20), 1467 (1982).

${ }^{28} \mathrm{~K}$. Ikeda and K. Matsumoto, "High-dimensional chaotic behavior in systems with time-delayed feedback," Phys. D 29(1), 223-235 (1987).

${ }^{29} \mathrm{~A}$. Neyer and E. Voges, "Dynamics of electrooptic bistable devices with delayed feedback," IEEE J. Quantum Electron. 18(12), 2009-2015 (1982).

${ }^{30}$ T. Erneux, L. Larger, M. W. Lee, and J.-P. Goedgebuer, "Ikeda hopf bifurcation revisited," Phys. D 194(1), 49-64 (2004).

${ }^{31}$ L. Larger, B. Penkovsky, and Y. Maistrenko, "Laser chimeras as a paradigm for multistable patterns in complex systems," Nat. Commun. 6, 7752 (2015).

${ }^{32}$ L. Larger, J.-P. Goedgebuer, and V. Udaltsov, "Ikeda-based nonlinear delayed dynamics for application to secure optical transmission systems using chaos," C. R. Phys. 5(6), 669-681 (2004).

${ }^{33}$ L. Illing and D. J. Gauthier, "Hopf bifurcations in time-delay systems with band-limited feedback," Phys. D 210(3), 180-202 (2005).

${ }^{34}$ K. E. Callan, L. Illing, Z. Gao, D. J. Gauthier, and E. Schöll, "Broadband chaos generated by an optoelectronic oscillator," Phys. Rev. Lett. 104(11), 113901 (2010).

${ }^{35}$ T. E. Murphy, A. B. Cohen, B. Ravoori, K. R. B. Schmitt, A. V. Setty, F. Sorrentino, C. R. S. Williams, E. Ott, and R. Roy, "Complex dynamics and synchronization of delayed-feedback nonlinear oscillators," Philos. Trans. R. Soc. London A 368(1911), 343-366 (2010).

${ }^{36}$ S. Heiligenthal, T. Dahms, S. Yanchuk, T. Jüngling, V. Flunkert, I. Kanter, E. Schöll, and W. Kinzel, "Strong and weak chaos in nonlinear networks with time-delayed couplings," Phys. Rev. Lett. 107, 234102 (2011).

${ }^{37}$ S. Heiligenthal, T. Jüngling, O. D'Huys, D. A. Arroyo-Almanza, M. C. Soriano, I. Fischer, I. Kanter, and W. Kinzel, "Strong and weak chaos in networks of semiconductor lasers with time-delayed couplings," Phys. Rev. E 88, 012902 (2013).

${ }^{38}$ M. Hermans, M. C. Soriano, J. Dambre, P. Bienstman, and I. Fischer, "Photonic delay systems as machine learning implementations," J. Mach. Learn. Res. 16, 2081 (2015).

${ }^{39}$ S. Ortín, M. C. Soriano, L. Pesquera, D. Brunner, D. San-Martín, I. Fischer, C. R. Mirasso, and J. M. Gutiérrez, "A unified framework for reservoir computing and extreme learning machines based on a single timedelayed neuron," Sci. Rep. 5, 14945 (2015). 